\title{
THE PACKING OF SPHERES IN THE SPACE $l_{p}$
}

\section{by JANE A. C. BURLAK, R. A. RANKIN and A. P. ROBERTSON}

(Received 4th June, 1958)

1. Introduction. A point $\mathbf{x}$ in the real or complex space $l_{p}$ is an infinite sequence $\left(x_{1}, x_{2}, x_{3}, \ldots\right)$ of real or complex numbers such that $\sum_{r=1}^{\infty}\left|x_{r}\right|^{p}$ is convergent. Here $p \geqslant 1$ and we write

$$
\|\mathbf{x}\|=\left\{\sum_{r=1}^{\infty}\left|x_{r}\right|^{p}\right\}^{1 / p}
$$

The unit sphere $S$ consists of all points $\mathbf{x} \in l_{p}$ for which $\|\mathbf{x}\| \leqslant 1$. The sphere of radius $a \geqslant 0$ and centre $\mathbf{y}$ is denoted by $S_{a}(\mathbf{y})$ and consists of all points $\mathbf{x} \in l_{v}$ such that $\|\mathbf{x}-\mathbf{y}\| \leqslant a$. The sphere $S_{a}(\mathbf{y})$ is contained in $S$ if and only if $\|\mathbf{y}\| \leqslant 1-a$, and the two spheres $S_{a}(\mathbf{y})$ and $S_{a}(\mathbf{z})$ do not overlap if and only if

$$
\|\mathbf{y}-\mathbf{z}\| \geqslant 2 a .
$$

The statement that a finite or infinite number of spheres $S_{a}(y)$ of fixed radius $a$ can be packed in $S$ means that each sphere $S_{a}(y)$ is contained in $S$ and that no two such spheres overlap.

In a recent paper [3] the packing of spheres $S_{a}(y)$ in $S$ was considered for the case $p=2$. It is the object of the present paper to extend this work to all $p \geqslant 1$. The results obtained are of $a$ different character according as $p \leqslant 2$ or $p>2$, and in the latter case are somewhat surprising.

We write

$$
\lambda_{p}=\left\{1+2^{1-1 / p}\right\}^{-1}, \quad \mu_{p}=\left\{1+2^{1 / p}\right\}^{-1} .
$$

Observe that $\lambda_{2}=\mu_{2}$ and that $\lambda_{p}<\mu_{p}$ when $p>2$. As usual, $\delta_{n r}$ is 1 or 0 according as $n=r$ or $n \neq r$.

TheоRem 1. If $1 \leqslant p \leqslant 2$, an infinity of spheres $S_{a}(\mathbf{y})$ of fixed radius a can be packed in $S$ if and only if $a \leqslant \lambda_{p}$. If (i) $a \leqslant \lambda_{p}$, the spheres may be centred at the points $\mathbf{y}_{n}=\left(y_{n 1}, y_{n 2}, y_{n 3}, \ldots\right)$, where $y_{n r}=(1-a) \delta_{n r}(n \geqslant 1, r \geqslant 1)$. If (ii) $\lambda_{p}<a \leqslant 1$, the maximum number of spheres $S_{a}(y)$ which can be packed in $S$ does not exceed $L_{p}(a)$, where $L_{1}(a)=1$, and

$$
L_{p}(a)=\left\{1-\frac{1}{2}\left(\frac{1-a}{a}\right)^{p /(p-1)}\right\}^{-1} \quad(1<p \leqslant 2) .
$$

Theorem 2. If $p>2$, an infinity of spheres $S_{a}(\mathbf{y})$ of fixed radius a can be packed in $S$ if and only if $a \leqslant \lambda_{p}$. If (i) $a \leqslant \lambda_{p}$, the spheres may be centred at the points $\mathbf{y}_{n}=\left(y_{n 1}, y_{n 2}, y_{n 3}, \ldots\right)$, where $y_{n r}=(1-a) \delta_{n r}(n \geqslant 1, r \geqslant 1)$. If (ii) $\lambda_{p}<a \leqslant \mu_{p}$, any finite number, no matter how large, of spheres $S_{a}(\mathbf{y})$ can be packed in $S$, but an infinite number cannot. If (iii) $\mu_{p}<a \leqslant 1$, the maximum number of spheres $S_{a}(\mathbf{y})$ which can be packed in $S$ does not exceed

$$
M_{p}(a)=\left\{1-\frac{1}{2}\left(\frac{1-a}{a}\right)^{p}\right\}^{-1} .
$$

2. The case $1 \leqslant p \leqslant 2$. Suppose that $m$ spheres $S_{a}\left(\mathbf{y}_{j}\right)(1 \leqslant j \leqslant m)$ can be packed in $S$, where $m \geqslant 1, a \leqslant 1$. By a simple extension of Lemma 1 of [2] from $n$-dimensional Euclidean space to $l_{p}$, we find that 


\section{PACKING OF SPHERES IN $l_{p}$}

$$
\sum_{j=1}^{m}\left\|\mathbf{y}_{j}\right\|^{p} \geqslant 2^{p-1} m^{2-p}(m-1)^{p-1} a^{p}
$$

where the right-hand side denotes zero when $m=p=1$. Hence, for at least one sphere $S_{a}\left(\mathbf{y}_{\jmath}\right)$,

$$
\left\|\mathbf{y}_{j}\right\| \geqslant a\left\{2\left(1-\frac{1}{m}\right)\right\}^{1-1 / p},
$$

and so

$$
1 \geqslant a+\left\|\mathrm{y}_{j}\right\| \geqslant a\left[1+\left\{2\left(1-\frac{1}{m}\right)\right\}^{1-1 / p}\right] .
$$

If infinitely many spheres $S_{a}(y)$ can be packed in $S$ it follows that $1 \geqslant a\left(1+2^{1-1 / p}\right)$; i.e. $a \leqslant \lambda_{v}$.

If $\lambda_{p}<u \leqslant 1$ and $1<p \leqslant 2$, we deduce from (1) that

$$
\frac{1}{m} \geqslant 1-\frac{1}{2}\left(\frac{1-a}{a}\right)^{p /(p-1)}
$$

and, since the right-hand side is positive, $m \leqslant L_{p}(a)$. If $p=1$, (1) shows that $m=1=L_{1}(a)$, because of the convention stated above; for otherwise we should have $a \leqslant \frac{1}{2}=\lambda_{1}$.

Finally, part (i) of Theorem 1 follows since, for $a \leqslant \lambda_{p}$ and $j \neq k$,

$$
\left\|\mathbf{y}_{j}-\mathbf{y}_{k}\right\|=2^{1 / p}(1-a) \geqslant 2 a,
$$

and $\left\|\mathbf{y}_{k}\right\|=\mathbf{l}-a$. Thus no two of the spheres $S_{u}\left(\mathbf{y}_{k}\right)$ overlap and they are all contained in $S$. This completes the proof of Theorem 1 .

3. The case $p>2$. Suppose that $m$ spheres $S_{a}\left(\mathbf{y}_{j}\right)(1 \leqslant j \leqslant m)$ can be packed in $S$, where $m \geqslant 1, a \leqslant 1$. By a simple extension of Lemma 2 of [1] (with $\beta=p, c_{j}=1$ ), we find that

$$
\sum_{j=1}^{m}\left\|\mathbf{y}_{j}\right\|^{p} \geqslant 2(m-1) a^{p},
$$

so that, for at least one sphere $S_{a}\left(\mathbf{y}_{j}\right)$,

$$
\left\|\mathbf{y}_{j}\right\| \geqslant a\left\{2\left(1-\frac{1}{m}\right)\right\}^{1 / p}
$$

Hence

$$
1 \geqslant a+\left\|y_{j}\right\| \geqslant a\left[1+\left\{2\left(1-\frac{1}{m}\right)\right\}^{1 / p}\right]
$$

from which we deduce that

$$
\frac{1}{m} \geqslant 1-\frac{1}{2}\left(\frac{1-a}{a}\right)^{p}
$$

The right-hand side of (2) is positive if $\mu_{p}<a \leqslant l$, so that we then obtain $m \leqslant M_{p}(a)$, which proves part (iii) of Theorem 2.

Part (i) of Theorem 2 can be proved as in §2. To prove part (ii) we suppose that $\lambda_{\mathfrak{p}}<a \leqslant \mu_{p}$ and take any positive integer $m$. For $1 \leqslant j \leqslant m$ and $n \geqslant l$ put

$$
y_{j n}=\varepsilon_{j n} 2^{(1-m) / p}(1-a)=\varepsilon_{j n} b \quad\left(n \leqslant 2^{m-1}\right), \quad y_{j n}=0 \quad\left(n>2^{m-1}\right),
$$

where $\varepsilon_{j n}$ is 1 or -1 according as the integral part of $(n-1) 2^{j-1 n}$ is even or odd. Then 
$\mathbf{y}_{j}=\left(y_{j 1}, y_{j 2}, y_{j 3}, \ldots\right) \in l_{p}$. For example, when $m=4$ the first 8 components of $\mathbf{y}_{1}, \mathbf{y}_{2}, \mathbf{y}_{3}$ and $\mathbf{y}_{4}$ are as follows :

$$
\begin{array}{rrrrrrrr}
b & b & b & b & b & b & b & b \\
b & b & b & b & -b & -b & -b & -b \\
b & b & -b & -b & b & b & -b & -b \\
b & -b & b & -b & b & -b & b & -b
\end{array}
$$

Clearly $\left\|\mathbf{y}_{j}\right\|=1-a$ for $1 \leqslant j \leqslant m$ and, for $1 \leqslant j<k \leqslant m$,

$$
\left\|\mathbf{y}_{j}-\mathbf{y}_{k}\right\|=2^{1-1 / p}(1-a) \geqslant 2 a \text {, }
$$

so that the $m$ spheres $S_{a}\left(\mathbf{y}_{j}\right)$ are packed in $S$. Since $m$ can be any positive integer this proves part (ii).

It remains to prove that if an infinity of spheres $S_{a}(y)$ can be packed in $S$, then $a \leqslant \lambda_{p}$. We therefore suppose that the spheres $S_{a}\left(\mathbf{y}_{n}\right)(n=1,2,3, \ldots)$ can be packed in $S$, where $\mathrm{y}_{n}=\left(y_{n 1}, y_{n 2}, y_{n 3}, \ldots\right)$. By considering each coordinate $y_{n r}(r=1,2,3, \ldots)$ in turn, picking out convergent subsequences, and renumbering the spheres, we may suppose that, for each fixed $r \geqslant 1, y_{n r} \rightarrow y_{r}$, say, as $n \rightarrow \infty$.

Since, for every positive integer $N$ and all $n \geqslant 1$,

$$
\sum_{r=1}^{N}\left|y_{n r}\right|^{p} \leqslant\left\|\mathbf{y}_{n}\right\|^{p} \leqslant(1-a)^{p}
$$

we have

$$
\sum_{r=1}^{N}\left|y_{r}\right|^{p} \leqslant(1-a)^{p}
$$

and it follows that $\mathrm{y}=\left(y_{1}, y_{2}, y_{3}, \ldots\right) \in l_{\mathrm{p}}$ and

$$
\|\mathbf{y}\| \leqslant \mathbf{l}-\boldsymbol{a} \text {. }
$$

Now take any positive integer $n$ and any $\varepsilon>0$ and choose an integer $N$, depending on $n$ and $\varepsilon$, such that

$$
\sum_{r>N}\left|y_{n r}\right|^{p}<\varepsilon^{p}
$$

Then, for $m>n$, since $S_{a}\left(\mathbf{y}_{m}\right)$ and $S_{a}\left(\mathbf{y}_{n}\right)$ do not overlap,

$$
\begin{aligned}
(2 a)^{p} & \leqslant \sum_{r=1}^{\infty}\left|y_{m r}-y_{n r}\right|^{p} \quad \ldots \ldots \ldots \ldots \ldots \ldots \ldots . . . . . . . \\
& =\sum_{r=1}^{N}\left|y_{m r}-y_{n r}\right|^{p}+\sum_{r>N}\left|y_{m r}-y_{n r}\right|^{p} .
\end{aligned}
$$

Now by Minkowski's inequality,

Hence, by (4),

$$
\begin{aligned}
\left\{\sum_{r>N}\left|y_{m r}-y_{n r}\right|^{p}\right\}^{1 / p} & \leqslant\left\{\sum_{r>N}\left|y_{m r}\right|^{p}\right\}^{1 / p}+\left(\sum_{r>N}\left|y_{n r}\right|^{p}\right\}^{1 / p} \\
& \leqslant(1-a)+\varepsilon .
\end{aligned}
$$

$$
(2 a)^{p}-(1-a+\varepsilon)^{p} \leqslant \sum_{r=1}^{N}\left|y_{m r}-y_{n r}\right|^{p}
$$


If we let $m \rightarrow \infty$ in this inequality, we obtain

$$
(2 a)^{p}-(1-a+\varepsilon)^{p} \leqslant \sum_{r=1}^{N}\left|y_{r}-y_{n r}\right|^{p} \leqslant \sum_{r=1}^{\infty}\left|y_{r}-y_{n r}\right|^{p}
$$

from which, since $\varepsilon$ is arbitrary, we deduce that

$$
(2 a)^{p}-(1-a)^{p} \leqslant \sum_{r=1}^{\infty}\left|y_{r}-y_{n r}\right|^{p}
$$

We now apply to (5) an argument similar to that applied to (3). For any $\varepsilon>0$, choose a positive integer $N$, depending on $\varepsilon$, such that

$$
\sum_{r>N}\left|y_{r}\right|^{p}<\varepsilon^{p}
$$

In place of (4) we have

$$
\begin{aligned}
(2 a)^{p}-(1-a)^{p} & \leqslant \sum_{r=1}^{N}\left|y_{r}-y_{n r}\right|^{p}+\sum_{r>N}\left|y_{r}-y_{n r}\right|^{p} \\
& \leqslant \sum_{r=1}^{N}\left|y_{r}-y_{n r}\right|^{p}+(1-a+\varepsilon)^{p} .
\end{aligned}
$$

On letting $n \rightarrow \infty$ we get, since $\varepsilon$ is arbitrary,

$$
(2 a)^{p} \leqslant 2(1-a)^{p}
$$

which is equivalent to $a \leqslant \lambda_{b}$. This completes the proof of Theorem 2 .

4. The case $p=\infty$. In this case $S$ can be interpreted as the "cube" consisting of points $\mathbf{X}=\left(x_{1}, x_{2}, x_{3}, \ldots\right)$ for which $\left|x_{r}\right| \leqslant 1$ for $r=1,2,3, \ldots$, and similarly for $S_{a}(\mathbf{y})$. It is clear that for $\frac{1}{2}<a \leqslant 1$, only one cube $S_{a}(y)$ can be packed in $S$, while, for $a \leqslant \frac{1}{2}$, infinitely many can ; for we may take their centres at the points $\left( \pm \frac{1}{2}, \pm \frac{1}{2}, \pm \frac{1}{2}, \ldots\right)$.

\section{REFERENCES}

1. R. A. Rankin, On sums of powers of linear forms I, Ann. of Math., 50 (1949), 691-698.

2. R. A. Rankin, On sums of powers of linear forms II, Ann. of Math., 50 (1949), 699-704.

3. R. A. Rankin, On packings of spheres in Hilbert space, Proc. Glasgow Math. Assoc., 2 (1955), 145-146.

\section{The UnIVERSITY}

Gr.Asgow 